\title{
Reversibility of primary pulmonary hypertension during six years of treatment with oral diazoxide
}

Sir,

We were interested to see the report by Chan et al (1987;57:207-9). In 1981 we described a patient with primary pulmonary hypertension who went into a complete remission after treatment with oral diazoxide. ${ }^{1}$ The drug was stopped after 15 months because of side effects and the pulmonary hypertension returned. Diazoxide was recommenced and the patient has remained well and symptom free since. It is now nine years since she originally presented. Although her pulmonary artery pressure has not been measured recently she is clinically in remission. There is slight elevation of the venous pressure and the right ventricle is easily palpable. There is a soft ejection systolic murmur in the pulmonary area and a loud pulmonary second sound. The chest radiographs show slight prominence of the main pulmonary artery but a normal heart size. There has been considerable improvement in the radiographic appearances since presentation and the electrocardiographic changes of right ventricular hypertrophy have resolved. Her only other treatment has been warfarin with chlorpropamide and diuretics for side effects of the diazoxide.
We are convinced that the improvement in our patient's condition is the result of diazoxide treatment and we intend to continue the drug indefinitely. The publication of this further case supports our view that vasodilator drugs should be considered in all patients with primary pulmonary hypertension.

D R Hall, Ipswich Hospital, Heath Road, Ipswich, Suffolk.

M C Petch, Papworth Hospital, Papworth Everard, Cambridge.

\section{Reference}

1 Hall DR, Petch MC. Remission of primary pulmonary hypertension during treatment with diazoxide. $\mathrm{Br}$ Med J 1981;282:1118.

\section{Viral myocarditis and dilated cardiomyopathy in early childhood}

Sir,

I read with interest the paper by Weinhouse et al (1986;56:94-7) describing the value of serial echocardiography in the follow up of infants and children with dilated cardiomyopathy. While agreeing with the value of such investigations, I remain concerned that the evidence for a viral myocarditis as the explanation of the dilated cardiomyopathy was somewhat incomplete.Viruses were grown from four of their 10 patients while in one an antibody titre suggested a recent infection. They indicated that all 10 infants had histories compatible with previous viral illnesses.

While I accept that it is very difficult to be certain of a viral aetiology in such patients, ${ }^{1}$ it is essential to exclude potentially treatable conditions. Other workers have emphasised the importance of looking carefully for an anomalous left coronary artery arising from the pulmonary trunk. ${ }^{23}$ Echocardiographic evidence and the use of other non-invasive techniques have at times proved inconclusive. Direct visualisation of the coronary arteries at aortography remains essential. Endomyocardial biopsy may sometimes provide information that is not obtainable in any other way. For example, we recently had a child admitted in severe cardiac failure, from whom a Coxsackie A virus was grown from a throat swab. She subsequently was shown on endomyocardial biopsy to have iron infiltration of 